\title{
Optimization of apparatus design and behavioral measures for the assessment of visuo-spatial learning and memory of mice on the Barnes maze
}

\author{
Timothy P. O'Leary and Richard E. Brown ${ }^{1}$ \\ Psychology and Neuroscience Department, Dalhousie University, Halifax, Nova Scotia, Canada, B3H 4 R2
}

\begin{abstract}
We have previously shown that apparatus design can affect visual-spatial cue use and memory performance of mice on the Barnes maze. The present experiment extends these findings by determining the optimal behavioral measures and test procedure for analyzing visuo-spatial learning and memory in three different Barnes maze designs. Male and female C57BL/6] mice were trained with a stable or random escape hole location and the sensitivities (statistical power) of four commonly used measures of learning and three measures of memory to detect differences between these training procedures were compared on each maze design. A maze design with a large diameter and no wall was optimal, because mice showed a reliable use of extra-maze visual cues, visuo-spatial search strategies, and spatial memory. A maze design with a small diameter, surrounding wall, and intra-maze visual cues was the least sensitive for determining visuo-spatial learning and memory, because mice showed little evidence of extra-maze cue use. Errors, distance traveled, and hole deviation scores were more sensitive measures of learning than latency to find the escape hole. Measures based on locating the escape hole (primary measures) were more sensitive than measures based on entering the escape hole (total measures). Measures of memory had similar levels of sensitivity on each maze. This experiment demonstrates that both apparatus design and the behavioral measures used as indicators of learning and memory can influence the ability of the Barnes maze to detect visuo-spatial learning and memory impairments in mice.
\end{abstract}

[Supplemental material is available for this article.]

The Barnes maze is a test of visuo-spatial learning and memory, originally designed for rats, which consists of an elevated circular platform with 18 holes located around the edge (Barnes 1979). Motivated to escape the bright lights and the open-space of the platform, rats search for an escape hole that leads to a dark box beneath the maze and, with training, they learn to use distal visual cues to determine the spatial location of the escape hole. In using the Barnes maze to test mice, however, the apparatus design and test procedures have often been modified from the original design validated for rats (Bach et al. 1995; Pompl et al. 1999; Miyakawa et al. 2001; Koopmans et al. 2003). In other tests of visuo-spatial learning and memory, such as the Morris water maze (MWM) and eight-arm radial maze, variations in apparatus design and test procedure have been found to influence learning and memory performance (Morris 1984; Gallagher et al. 1993; Crusio 1999; Levin 2001; Iivonen et al. 2003; Blokland et al. 2004; Wenk 2004; Crusio and Schwegler 2005; Van Dam et al. 2006; Maei et al. 2009; Wahlsten 2011) and we have found that modifications to the Barnes maze design, such as decreasing the diameter of the maze and adding a wall around the edge, reduced distal cue use and impaired visuo-spatial memory in mice (O'Leary et al. 2011; O'Leary and Brown 2012).

Given that variations in apparatus design can affect visuospatial cue use in mice, they should also influence the sensitivity to detect the effects of experimental manipulations on visuospatial learning and memory in the Barnes maze. For example, the Barnes maze has been found to be less sensitive than the

\footnotetext{
${ }^{1}$ Corresponding author

E-mail rebrown@dal.ca

Article is online at http://www.learnmem.org/cgi/doi/10.1101/Im.028076.112.
}

MWM and radial arm water maze for detecting visuo-spatial impairment in the Tg2576 mouse model of Alzheimer's disease (Stewart et al. 2011). Furthermore, performance differences between inbred strains on the Barnes maze are not always replicable across studies (Holmes et al. 2002; Nguyen et al. 2002; O'Leary et al. 2011), and on some designs blind mice can perform as well as sighted mice can, indicating that visual cues are not being used (Garcia et al. 2004; O'Leary et al. 2011; Yassine et al. 2013). These problems may be due to the use of suboptimal apparatus designs or procedures to test mice on the Barnes maze.

Despite its frequent use, the optimal apparatus design and test procedure for the assessment of visuo-spatial learning and memory of mice on the Barnes maze has not yet been determined. The optimal apparatus design and test procedure should provide valid and reliable measurements of visuo-spatial learning and memory and be sensitive enough to detect differences in performance with relatively small sample sizes. Validity of the Barnes maze as a visuo-spatial test can be established by demonstrating that performance is dependent on the use of extra-maze visuospatial cues (Barnes 1979). Reliability can be demonstrated by consistently detecting differences in performance between groups of mice following experimental manipulations at different times in the same laboratory and in different laboratories (Crabbe et al. 1999; Wahlsten et al. 2003). Sensitivity can be determined by examining how variations in experimental parameters influence the ability to detect visuo-spatial learning and memory impairments. This ability can be estimated by comparing the degree to which different measures of learning and memory performance discriminate between mice that are able to use visuospatial cues and mice that are not able to use such cues. Mice may fail to use visuo-spatial cues because of learning and memory 
impairments, visual impairments, or extra-maze cues not being available, or because the extra-maze visuo-spatial cues do not predict the spatial location of the escape hole.

Sensitivity to detect learning and/or memory impairments can also depend on the measure of performance used (Cahill et al. 2001). Although latency to find the escape hole is the most commonly used measure of learning in maze studies, it can be confounded by movement disorders or immobility (Gage et al. 1984; Gallagher et al. 1993; Alberto et al. 1999). Thus, performance measures which do not rely on speed of locomotion, such as the number of errors, distance traveled, and search path, have been used to analyze learning on the Barnes maze. Although Pompl et al. (1999) found that measures of total latency and errors were sensitive enough to detect learning impairment on the Barnes maze, other researchers have found that these measures have poor sensitivity for detecting differences between groups (Garcia et al. 2004; Harrison et al. 2006; O'Leary et al. 2011), while the sensitivities of other measures of learning and memory have yet to be evaluated.

Based on our previous research (O'Leary et al. 2011; O'Leary and Brown 2012), the present experiment was conducted (1) to determine the sensitivities of three different Barnes maze designs for detecting impairments in visuo-spatial learning and memory; (2) to determine the most sensitive measures of learning and memory for each maze design; and (3) to determine the reliability of our previous results (O'Leary and Brown 2012) on the validity of each maze design as tests of visuo-spatial learning and memory. Mice completed acquisition and reversal training to assess learning, and probe trials to assess memory on three different Barnes maze designs: (1) a maze design with a small diameter, wall, and intramaze cues (SWBM); (2) a design with a small diameter and no wall (SBM); and (3) a design with a large diameter and no wall (LBM). Half of the mice were trained with an escape box located in a stable location which was predicted by visuo-spatial cues, and the other half were trained with an escape box located at a random location, which could not be predicted by extra-maze cues. The sensitivities of four measures (latency, errors, hole deviation, and distance traveled) to detect differences in the rate of learning between groups were compared, along with the sensitivities of three measures (zone time, hole visits, and average proximity) to detect differences in memory. We also compared two ways of measuring learning performance: (1) latency, errors, and distance to first locate the escape hole (primary data) and (2) latency, errors, and distance to enter the escape hole (total data) (Harrison et al. 2006). Finally, the validity of each maze as a test of visuo-spatial learning was compared by examining the search strategies used to locate the escape hole and the size of the impairments in the reversal test. The performance in probe trials with extra-maze cues blocked by a cur- tain was used to examine the degree to which mice were using the extra-maze visual cues to remember the escape hole location.

\section{Results}

Data presented here are for primary measures of learning performance. After locating the escape hole, mice quickly entered the escape box (mean $<10 \mathrm{sec}$ ) on all three mazes after $5 \mathrm{~d}$ of training, and the results for the total latency, errors, and distance traveled are presented in the Supplemental results (Supplemental Figs. $1-3)$.

\section{Acquisition training}

Mice trained with a stable escape hole location found the escape hole significantly faster than mice trained with a random escape hole location in the $\operatorname{LBM}\left(F_{(1,22)}=7.36, P<0.05, \eta^{2}=0.25\right.$, $P w=0.74)$, but not on the SWBM, while the difference on the SBM just failed to reach significance $\left(F_{(1,22)}=3.82, P=0.06\right.$, $\left.\eta^{2}=0.15, P w=0.46\right)$ (Fig. 1A-C). Latency decreased significantly over days A1-A15 in all three mazes $(P<0.0001)$. Analysis of
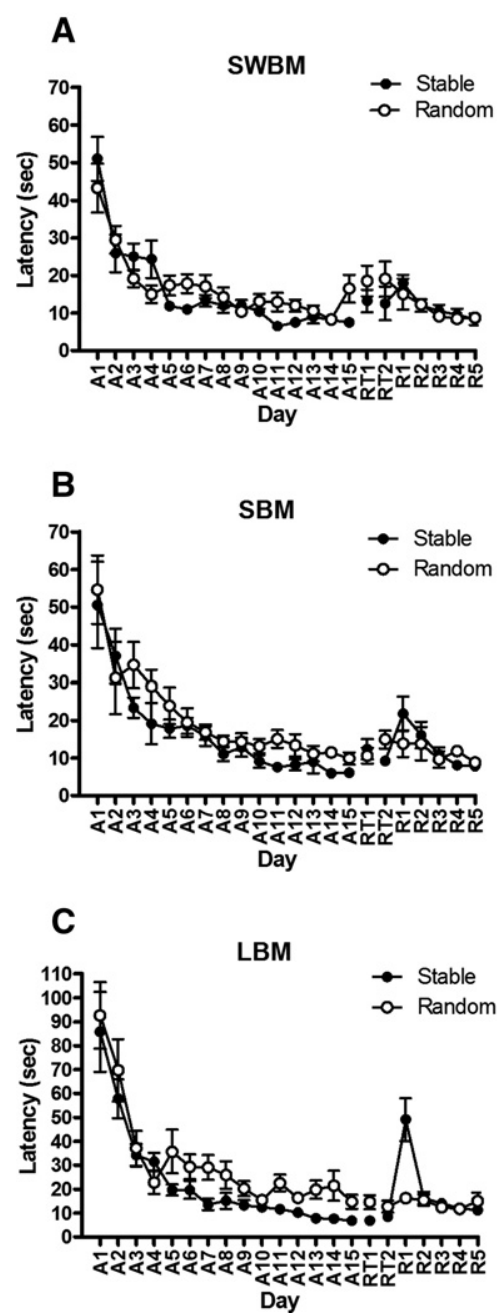

Figure 1. Mean ( \pm SEM) primary latency (sec) and number of errors to locate the escape hole in mice trained with a stable or random escape hole location during acquisition training (A1-A15), re-training days following probe trials (RT1 and RT2), and reversal training (R1-R5) on the SWBM $(A, D)$, the SBM $(B, E)$, and the $\operatorname{LBM}(C, F)$. 


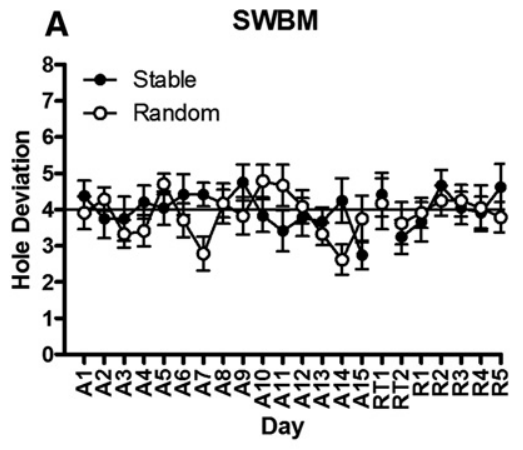

B
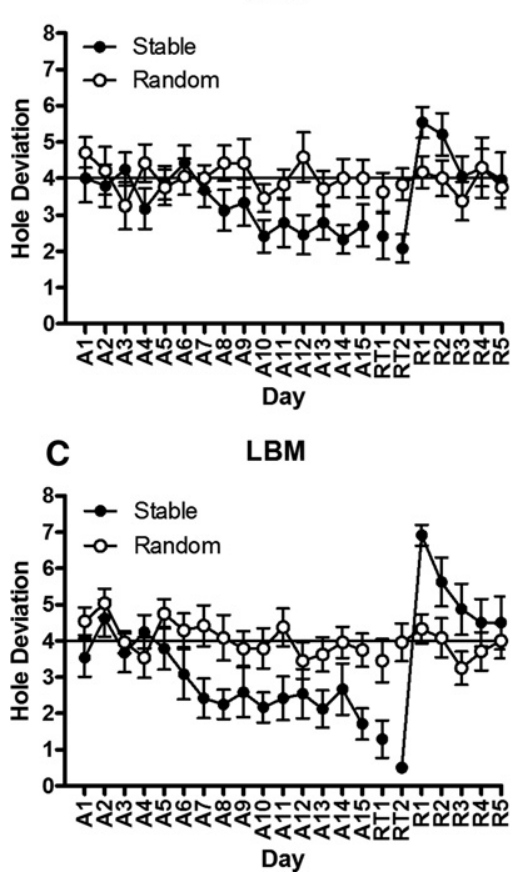

D

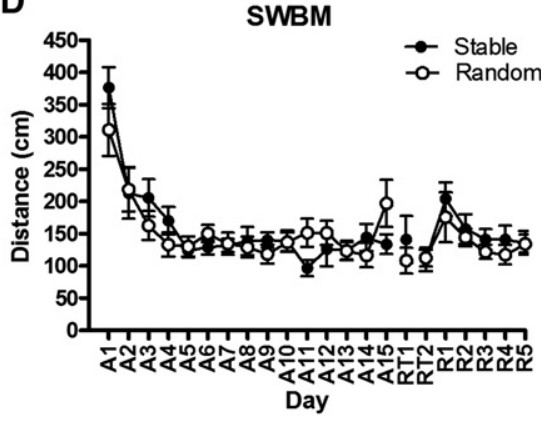

E

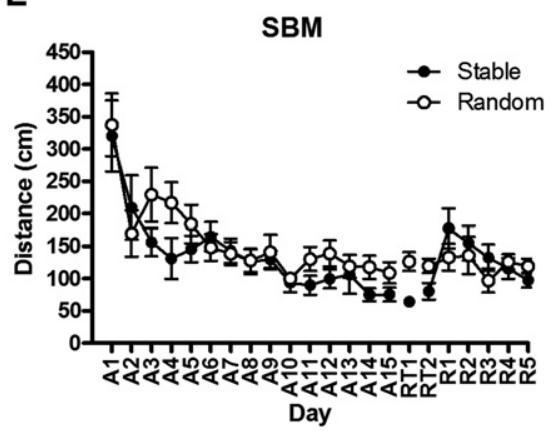

$\mathbf{F}$

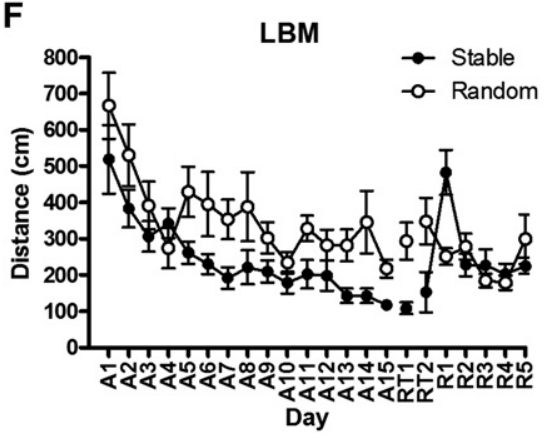

Figure 2. Mean ( \pm SEM) hole deviation scores (number of holes) and primary distance traveled $(\mathrm{cm})$ by mice trained with stable or random escape hole locations during acquisition training (A1-A15), retraining days following probe trials (RT1 and RT2), and reversal training (R1-R5) on the SWBM $(A, D)$, the $\operatorname{SBM}(B, E)$, and the $\operatorname{LBM}(C, F)$. The horizontal line indicates chance performance (four holes).

effect size as a function of training showed that mice with a stable escape hole location performed significantly better than mice trained with a random escape hole location in the LBM after $10 \mathrm{~d}$ of training (Fig. 3A, see below).

Mice trained with a stable escape hole location made fewer errors than mice trained with a random escape hole location in the SBM $\left(F_{(1,22)}=12.03, P<0.005, \eta^{2}=0.35, P w=0.91\right)$ and the $\operatorname{LBM}\left(F_{(1,22)}=44.96, P<0.0001, \eta^{2}=0.67, P w=0.99\right)$, but did not differ significantly on the $\operatorname{SWBM}\left(F_{(1,22)}=3.84, P<\right.$ $0.063, \eta^{2}=0.15, P w=0.47$ ) (Fig. 1D-F). Errors decreased significantly over days A1-A15 on all three mazes $(P<0.0001)$. Analysis of effect size as a function of training showed that mice with a stable escape hole location performed significantly better than mice with a random escape hole location after $6 \mathrm{~d}$ of training on the LBM and $9 \mathrm{~d}$ of training on the SBM (Fig. 3B, see below).

Mice trained with a stable escape hole location had significantly smaller hole deviation scores than mice trained with a random escape hole location on the SBM $\left(F_{(1,22)}=8.28, P<0.01\right.$, $\left.\eta^{2}=0.27, P w=0.78\right)$ and the $\operatorname{LBM}\left(F_{(1,22)}=25.66, P<0.0001\right.$, $\left.\eta^{2}=0.54, P w=0.99\right)$, but not on the SWBM (Fig. 2A-C). One-way ANOVAs showed that mice trained with a stable escape hole location decreased their hole deviation scores across days on the $\operatorname{LBM}\left(F_{(14,154)}=2.75, P<0.005\right)$ and the SBM $\left(F_{(14,154)}=1.95, P<0.05\right)$, while mice trained with a random escape hole location did not show a decrease in hole deviation scores on these mazes (Fig. 2B,C). On the SWBM, mice trained with a random escape hole location showed differences in hole deviation scores over days $\left(F_{(14,308)}=1.85, P<0.05\right)$, but mice trained with a stable escape hole location did not (Fig. 2A). Analysis of effect size as a function of training showed that mice with a stable escape hole location performed consistently better than mice with a random escape hole location after $7 \mathrm{~d}$ of training on the LBM and $12 \mathrm{~d}$ on the SBM (Fig. 3C).

Mice trained with a stable escape hole location traveled shorter distances before locating the escape hole than mice trained with a random escape hole location on the SBM $\left(F_{(1,22)}=4.72, P<\right.$ $\left.0.05, \eta^{2}=0.18, P w=0.55\right)$ and the LBM $\left(F_{(1,22)}=34.54, \quad P<0.0001, \quad \eta^{2}=0.61\right.$, $P w=0.99$ ), but not on the SWBM (Fig. 2D-F). Distance traveled decreased significantly over days A1-A15 on all three mazes $(P<0.0001)$. Analysis of effect size as a function of training showed that mice trained with a stable escape hole performed consistently better than mice trained with a random escape hole location after $5 \mathrm{~d}$ on the LBM and $14 \mathrm{~d}$ on the SBM (Fig. 3D).

\section{Search strategy use}

In the SWBM, mice trained with a stable escape hole used the random search strategy on the first block, but infrequently thereafter $\quad\left(\chi^{2}(4)=19.45, \quad P<0.0001\right)$ (Fig. 4A). They used the serial search strategy more than the spatial and random strategies on all five training blocks $(P<0.05)$. Use of the spatial search strategy did not increase with training and was used less than expected by chance. Mice trained in the SWBM with a random escape hole (Fig. 4B) used the random and the serial search strategies equally often on the first block of trials, and use of the random search strategy decreased over blocks $\left(\chi^{2}(4)=13.87, P<0.01\right)$, while use of the serial search strategy increased $\left(\chi^{2}(4)=14.60, P<0.001\right)$ (Fig. 4B). The serial strategy was used more than the random strategy on blocks $2-5$ $(P<0.01)$, and more than the spatial strategy on all training blocks $(P<0.005)$.

In the SBM, mice trained with a stable escape hole used the random strategy significantly more than the serial strategy on the first training block $(P<0.05)$ (Fig. 4C). Use of the random search strategy decreased across training blocks $\left(\chi^{2}(4)=18.28\right.$, $P<0.005)$. Both the serial $\left(\chi^{2}(4)=11.12, P<0.05\right)$ and spatial strategies increased over training blocks $\left(\chi^{2}(4)=11.72, P<\right.$ $0.05)$, and although the serial strategy was used the most on blocks 2 and $3(P<0.05)$, the spatial strategy was used the most 

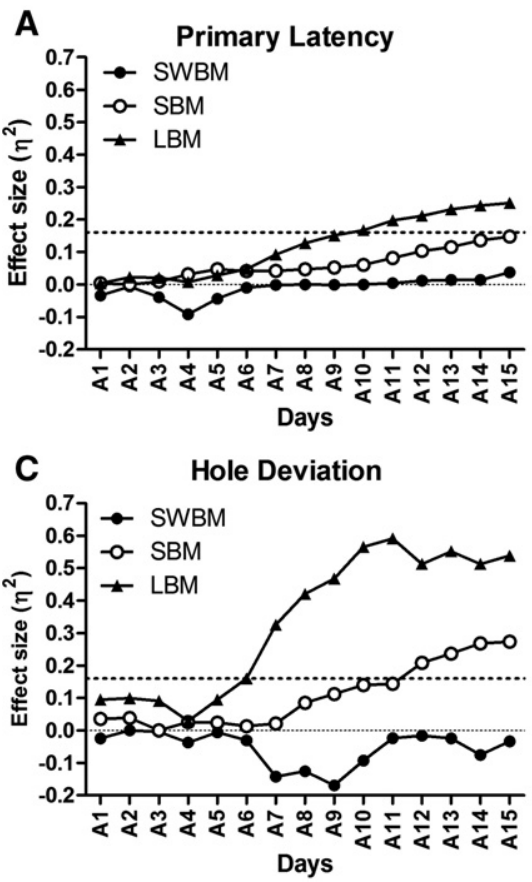
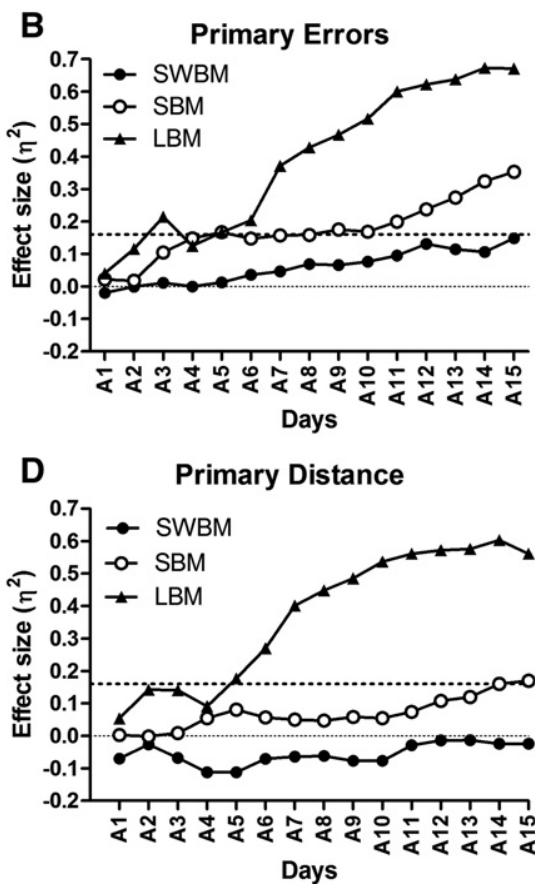

Figure 3. Effect size $\left(\eta^{2}\right)$ displayed as a function of the cumulative number of training days for primary latency to enter the escape hole $(A)$, errors $(B)$, hole deviation scores $(C)$, and distance traveled $(D)$. The dashed line represents the effect size required for a significant effect of hole location $(\sim 0.163)$ given $N=12$ mice per group. Effect sizes are negative when mice trained with a random escape hole location performed better than mice trained with a stable escape hole location.

on block 5. Mice trained in the SBM with a random escape hole location used the random and the serial search strategies equally often on the first block of trials. Over blocks $2-5$, however, use of the random search strategy decreased $\left(\chi^{2}(4)=25.78, P<0.0001\right)$, while use of the serial search strategy increased $\left(\chi^{2}(4)=12.52\right.$, $P<0.05$ ) (Fig. 4D). The serial search strategy was used more than the random strategy and the spatial strategy on blocks $2-5$ $(P<0.05)$. Use of the spatial search strategy did not increase significantly over training.

In the LBM, mice trained with a stable escape hole used the random strategy more than the spatial strategy on the first training block $(P<0.005)$ (Fig. 4E). Use of the random search strategy decreased across training blocks $\left(\chi^{2}(4)=29.88, P<0.0001\right)$, and use of the serial search strategy peaked on block 2 . Use of the spatial strategy increased over the training blocks $\left(\chi^{2}(4)=20.55, P<\right.$ $0.0005)$, and was used most often on blocks 4 and $5(P<0.05)$. Mice trained in the LBM with a random escape hole used the random search strategy more than the other strategies on the first block $(P<0.05)$, but then use of the random search strategy decreased $\left(\chi^{2}(4)=24.67, P<0.0001\right)$, while use of the serial search strategy increased $\left(\chi^{2}(4)=19.88, P<0.001\right)$ (Fig. $4 \mathrm{~F}$ ) and was used more than the random or spatial strategies on blocks $2-5$ $(P<0.05)$.

\section{Acquisition probe trials}

In the SWBM (Fig. 5A) neither the mice trained with a stable escape hole location, nor those trained with a random escape hole location showed an increase in time in the correct zone in the visually cued probe trial. In both the $\operatorname{SBM}\left(F_{(1,22)}=8.90, P<\right.$ $\left.0.01, \eta^{2}=0.29, P w=0.83\right)$ and $\operatorname{LBM}\left(F_{(1,22)}=40.66, P<0.0001\right.$, $\left.\eta^{2}=0.65, P w=0.99\right)$, mice trained with a stable escape hole location spent more time in the correct zone than mice trained with a a smaller average proximity to the correct hole than expected by chance on any of the three maze designs.

In the curtain probe trial, when visual cues were not available, mice trained with a stable escape hole location did not differ from mice trained with a random escape hole location in the time spent in the correct zone on any maze design (Fig. 6A). Mice trained with a stable escape hole location did not spend more time than expected by chance in any of the zones on any of the maze designs. Mice trained with a random escape hole location did not spend more time than expected by chance in any of the zones on the SBM and SWBM (Fig. 6B).

\section{Reversal effects: Latency, errors, hole deviation, and distance traveled}

Given that primary measures of performance were nearly identical to those of total measures during reversal training, only the primary measures were analyzed. Mice trained with a stable escape hole location on the LBM had larger reversal effects (R1 and RT2) than mice trained with a random escape hole location for latency $\left(F_{(1,22)}=21.40, P<0.0005\right)$, errors $\left(F_{(1,22)}=19.87\right.$, $P<0.0005)$, hole deviation scores $\left(F_{(1,22)}=49.17, P<0.0001\right)$, and distance traveled $\left(F_{(1,22)}=13.59, P<0.005\right)$, whereas significant differences on the SBM were present only for hole deviation scores $\left(F_{(1,22)}=11.12, P<0.005\right)$, and no significant differences were found on the SWBM (Fig. 7A-D). Reversal effects were significantly greater than zero for mice trained with a stable escape hole on the LBM for all four measures of learning, while on the SBM reversal effects were significantly greater than zero for measures of latency, distance, and hole deviation. On the SWBM reversal effects differed significantly from zero for latency, errors, and distance. Reversal effects did not differ 

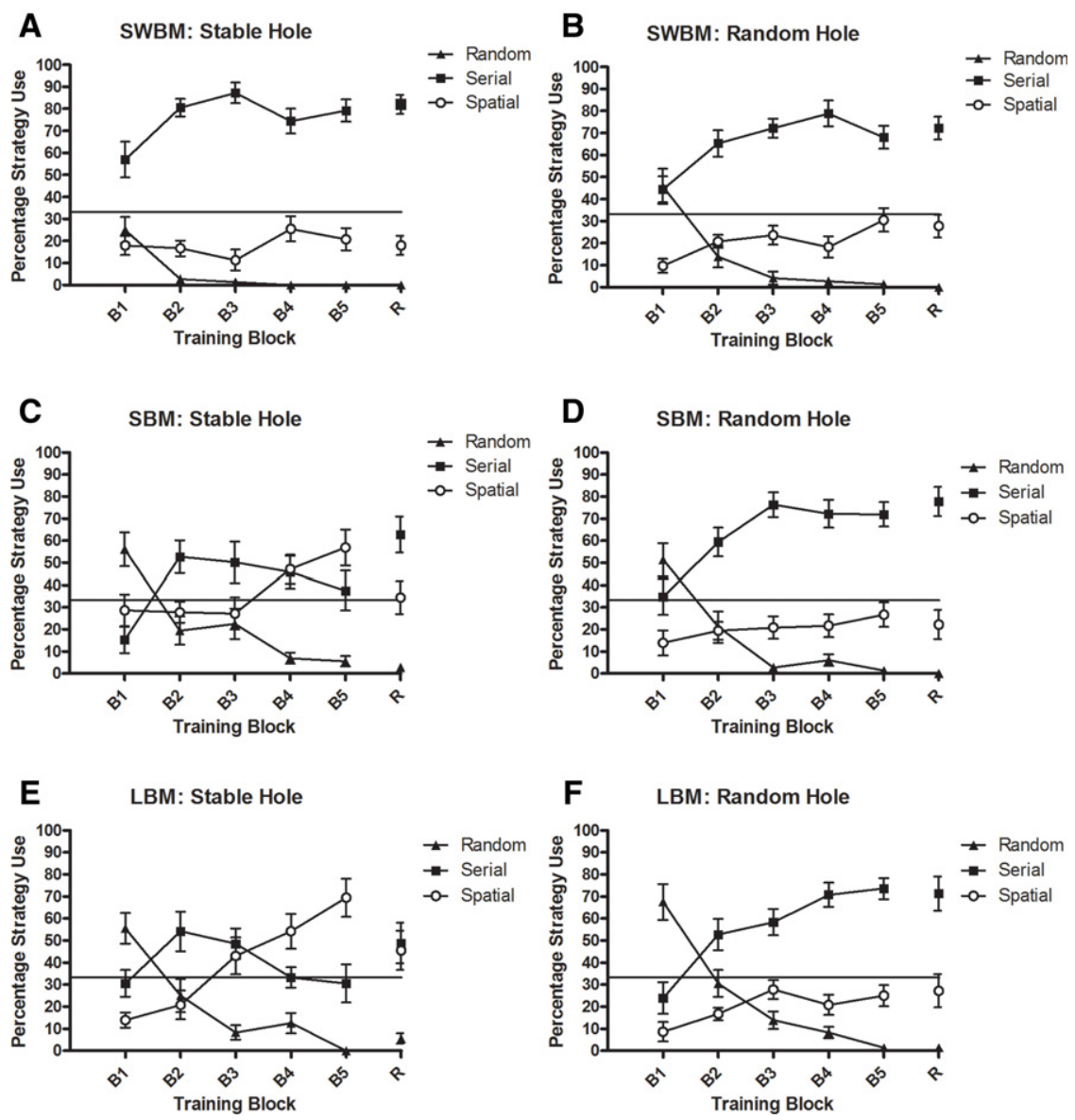

Figure 4. Percentage of trials that the random, serial, and spatial search strategies were used to locate the escape hole on six-trial training blocks during acquisition training (B1-B5) and reversal training (R) for mice trained with a stable or random escape hole on the SWBM $(A, B)$, the SBM $(C, D)$, and the LBM $(E, F)$. The horizontal line indicates the chance level of performance $(33.33 \%)$ given equal use of all three strategies.

from zero for mice trained with a random escape hole location for any of the measures of learning, on any of the mazes.

\section{Reversal training}

During reversal training (days R1-R5) mice with a stable escape hole location in the SWBM did not differ from mice trained with a random escape hole location on any measure of learning (Figs. 1A,D, 2A,D). Over days R1-R5, both groups tested in the SWBM showed a decline in latency to locate the escape hole $\left(F_{(2.03,44.64)}=5.72, P<0.01\right)$, number of errors $\left(F_{(2.31,50.73)}=\right.$ $3.76, P<0.05)$, and a marginally significant trend in distance traveled $\left(F_{(2.34,51.38)}=2.87, P=0.058\right)$, but not in hole deviation scores. On the SBM, mice trained with a stable escape hole location did not differ from those trained with a random escape hole on any measure of learning (Figs. 1B,E, 2B,E). Over days R1-R5 there was a decrease in latency to find the escape hole $\left(F_{(2.11,46.49)}=4.89, P<0.05\right) \quad$ (Fig. 1B) and number of errors $\left(F_{(2.54,55.85)}=3.73, P<0.05\right)$ (Fig. $\left.1 \mathrm{E}\right)$, but not distance traveled (Fig. 2E) or hole deviation scores (Fig. 2B).

In the LBM, mice trained with a stable escape hole location were significantly slower to locate the escape hole $\left(F_{(1,22)}=6.57\right.$, $P<0.05$ ) (Fig. 1C) and had larger hole deviation scores $\left(F_{(1,22)}=\right.$ 12.85, $P<0.005$ ) (Fig. 2C) than mice trained with a random es- cape hole location, but no differences were found in distance traveled or errors (Figs. 1F, 2F). Differences in latency $\left(F_{(1.68,36.91)}=10.07, P<0.001\right)$, distance traveled $\left(F_{(4,88)}=4.77, P<0.005\right)$, and errors $\quad\left(F_{(2.83,62.32)}=5.99, \quad P<0.005\right)$ were present on day R1, but not on other reversal days. On the LBM there were significant decreases over days R1R5 in latency $\left(F_{(1.68,36.91)}=13.46, P<\right.$ $0.0001)$, number of errors $\left(F_{(2.83,62.32)}=\right.$ 7.53, $P<0.0005)$, hole deviation scores $\left(F_{(3.03,66.64)}=2.95, \quad P<0.05\right)$, and distance traveled to find the escape hole $\left(F_{(4,88)}=6.32, P<0.0005\right)$.

During reversal training, mice trained with a stable escape hole on the SBM and LBM reverted to a serial search strategy, and by the end of reversal training only mice in the LBM used the spatial strategy more than chance (Fig. 4). This indicates that there were not enough trials in the reversal phase for mice on the SBM and LBM to develop a new spatial search strategy. Thus, their performance generally did not differ from the mice trained with a random hole location because they both used the serial search strategy (see Figs. 1, 2).

\section{Reversal probe trial}

Since percentage time in each zone, number of hole visits, and proximity to the correct hole had similar levels of sensitivity, we analyzed data only for the percentage time in each zone in the reversal probe trial. In the SWBM, mice trained with a stable escape hole location did not spend more time in the correct zone than mice trained with a random escape hole location, and neither group spent more time than expected by chance in any zone (Fig. 8A). In the SBM, mice trained with a stable escape hole location spent more time in the correct zone than mice trained with a random escape hole location $\left(F_{(1,22)}=12.71, P<0.005\right)$, and they spent more time in $\mathrm{ZC}, \mathrm{Z15}$, and $\mathrm{Z} 9$ than expected by chance, indicating that they remembered the location of the reversal escape hole (Fig. 8B) and the location of the escape hole during acquisition training. Mice trained with a random escape hole location in the SBM did not spend more time than expected by chance in any zone. In the LBM, mice trained with a stable escape hole location spent more time in the correct zone than mice trained with a random escape hole location $\left(F_{(1,22)}=11.46, P<0.005\right)$ (Fig. $8 \mathrm{C}$ ). Mice trained with the stable escape hole location spent more time than expected by chance in the correct zone (ZC) and near the old acquisition escape hole (Z8). Mice trained with the random escape hole location only spent more time in Z14 than expected by chance.

\section{Discussion}

The results of the present experiment provide answers to eight questions about the optimal apparatus design and test procedure for the assessment of visuo-spatial learning and memory of mice on the Barnes maze. 

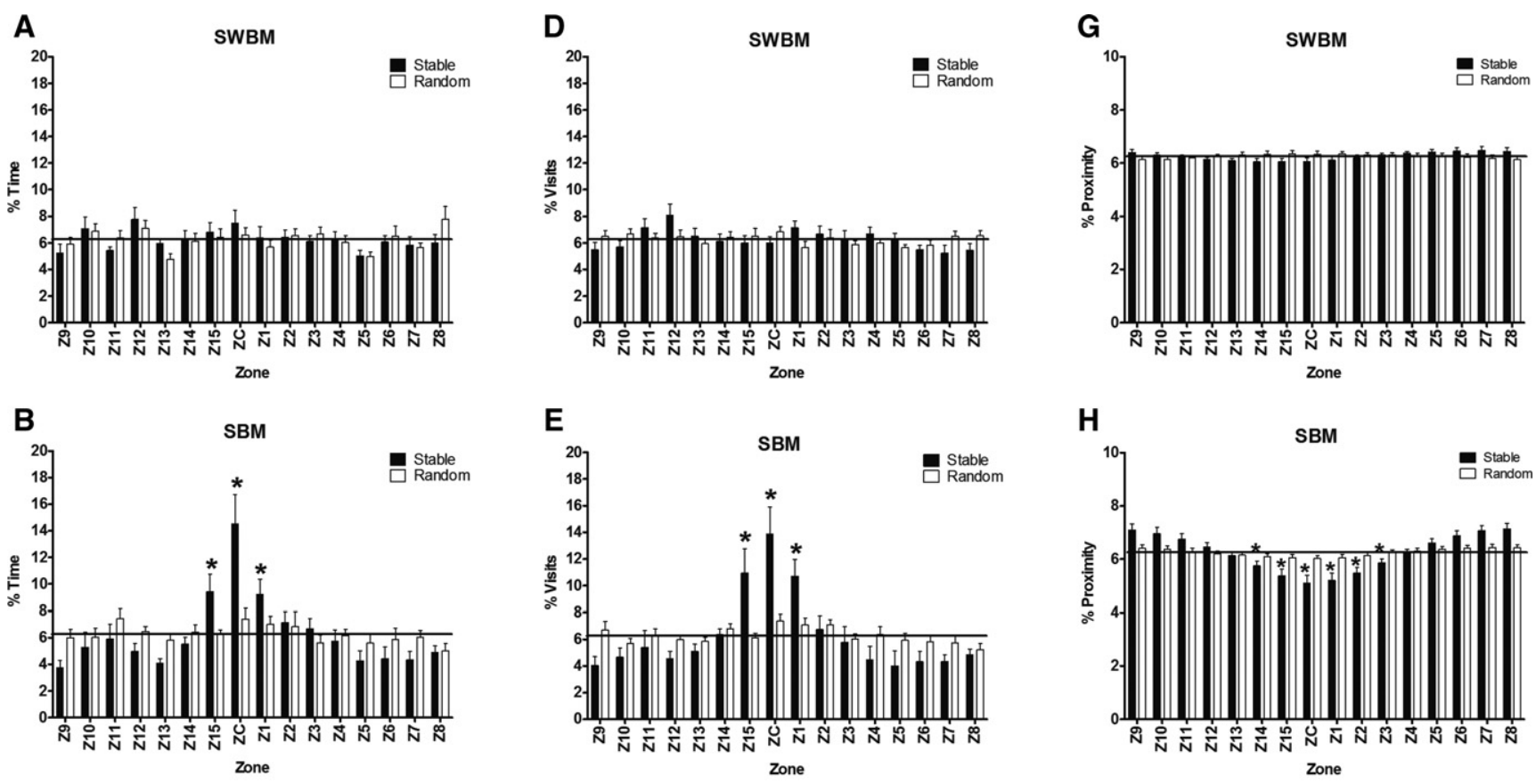

E

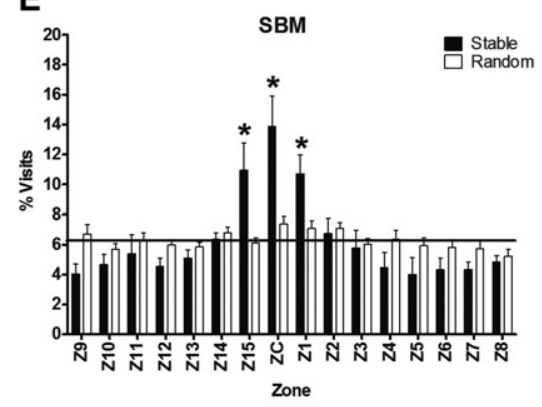

$\mathbf{F}$

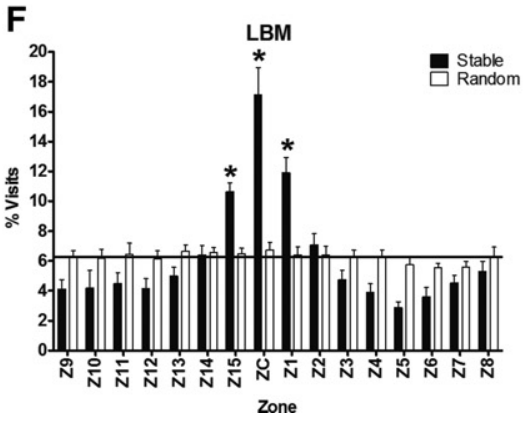

H
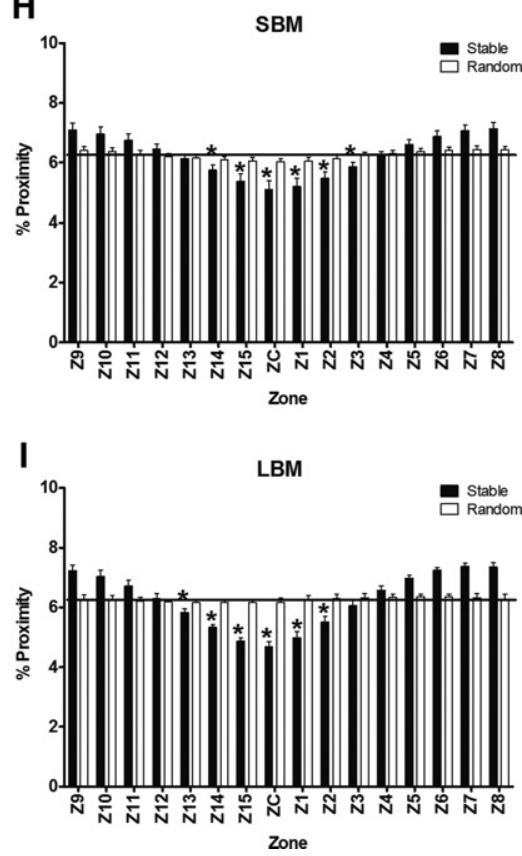

Figure 5. Percentage of time ( + SEM), hole visits, and average proximity (distance in centimeters) to the correct zone (ZC) and the other 15 zones (Z1 Z15) of the maze during the acquisition probe trial with visible extra/intra-maze cues for mice trained with a stable or random escape hole location on the $\operatorname{SWBM}(A, D, G)$, the $\operatorname{SBM}(B, E, H)$, and the $\operatorname{LBM}(C, F, I)$. The horizontal line indicates the chance level of performance $(6.25$ sec $)$ and the asterisks $\left({ }^{*}\right)$ indicate when percentage time, hole visits, or average proximity are greater than expected by chance $(P<0.05)$. Note that performance is better as the average proximity away from the correct hole decreases.

How does apparatus design influence the sensitivity to detect impairments in visuo-spatial learning and memory in the Barnes maze?

The sensitivity to detect differences in performance of mice trained with stable and random escape hole locations was poor in the SWBM because mice used the nonspatial serial search strategy when trained with either a stable or random escape hole location. The sensitivity for detecting differences in visuo-spatial learning and memory performance was higher on the SBM, because mice trained with a stable escape hole performed better than mice trained with a random escape hole on all measures of learning except latency to locate the escape hole, and showed better memory performance during both acquisition and reversal probe trials. The sensitivity for detecting differences in visuospatial learning and memory performance was highest on the LBM, because mice trained with a stable escape hole performed better than mice trained with a random escape hole on all measures of learning and memory performance.

The variability in sensitivity to detect differences in learning and memory performance between apparatus designs was due to differences in ability to associate visual cues with the escape hole location. Mice may find it easier to use visual cues to locate the escape hole on the LBM than on the SWBM or the SBM due to the LBM's increased size. On the LBM, mice are able to correct their search path before reaching the edge of the maze during acquisition training, therefore improving performance measures of learning. Furthermore, the increased distance between escape holes on the LBM allows the association between visual cues and the escape hole to be less precise, and yet still allow for accurate discrimination between the spatial locations of different holes. Increasing the diameter of the maze also decreased the efficiency of the serial search strategy, promoting the use of extra-maze visual cues and the use of the spatial search strategy on the LBM.

Which measures of performance are the most sensitive to detect impairments in visuo-spatial learning on the Barnes maze?

The latency to locate the escape hole, perhaps the most widely used measure of learning, was the least sensitive for detecting 

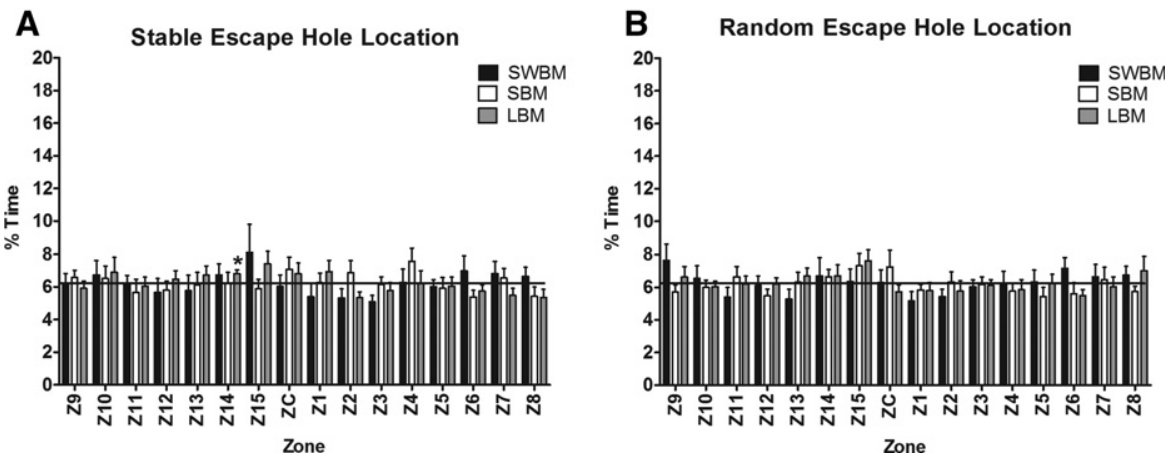

Figure 6. Percentage of time ( + SEM) in the correct zone $(Z C)$ and 15 other zones $(Z 1-Z 15)$ of the maze during the acquisition probe trial with a curtain for mice trained with stable $(A)$ or random $(B)$ escape hole locations on the SWBM, the SBM, and the LBM. The horizontal line indicates the chance level of performance $(6.25 \%)$, and the asterisk $\left({ }^{*}\right)$ indicates when visits to a zone were greater than expected by chance $(P<0.05)$.

differences between mice trained with stable and random escape hole locations. The number of errors, hole deviation scores, and distance traveled measures were more sensitive on the SBM and LBM, and we recommend that these measures be used in addition to latency to quantify learning in mice on the Barnes maze.

During the first $5 \mathrm{~d}$ of acquisition training on all three mazes, some mice did not immediately enter the escape hole once it was found, and thus primary latency, errors, hole-deviation, and distance measures of learning were used to control for delays in entering the escape hole. In general, the primary measures had greater sensitivity compared to that of the total measures, which resulted from a reduction of within-group error variance in performance which was not explicable by visuo-spatial ability. Alternatively, despite the random assignment of mice to training conditions, pre-existing differences may have been present between mice trained with a stable escape hole and mice trained with a random escape hole in the willingness to enter the escape hole, which would reduce the performance difference between training conditions using total measures of learning performance. Harrison et al. (2006) first demonstrated the increased sensitivity of primary measures, and found that total measures did not have sufficient sensitivity to detect performance differences between mice trained with stable and random escape hole locations. Our data, however, indicate that the increase in sensitivity for primary measures over total measures on the SBM and LBM was modest, and the discrepancy between our results and those of Harrison et al. (2006) may be due to differences in apparatus design and/or test procedures. We suspect that the use of a larger maze $(120 \mathrm{~cm}$ vs. $90 \mathrm{~cm})$ and the addition of bright lights and a loud buzzer in the present study promoted the use of the spatial search strategy and reduced the tendency for mice to explore the maze after locating the escape hole, thus increasing the sensitivity of the total measures of performance.

Examination of acquisition rates showed that mice trained with stable and random escape hole locations on the SBM and LBM improved their perfor-
A

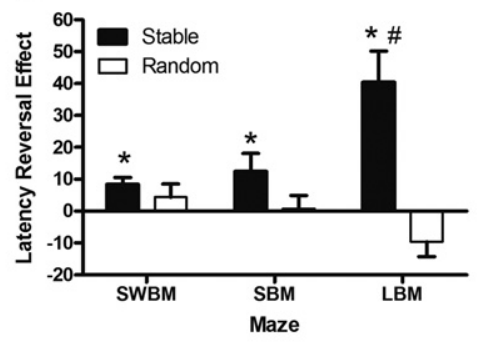

C

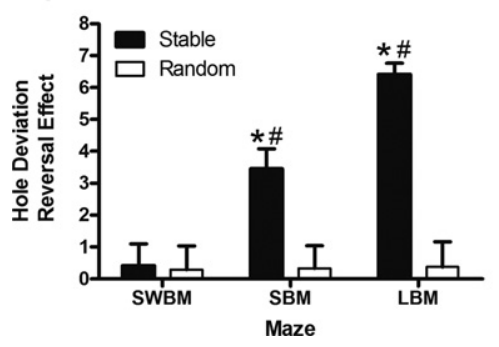

mance with training, and the effect sizes for differences in the training procedure increased as a function of training days. On the LBM, mice trained with a stable escape hole required a minimum of 1020 trials (5-10 d) before they performed consistently better than mice trained with a random escape hole on each measure of learning (Fig. 1B). The increase in effect size over days in the SBM and LBM acquisition data reflects the increased use of the spatial search strategy over training days. The greatest effect sizes did not occur until mice with the stable escape hole location were predominately using the spatial search strategies on blocks 4 and 5 (Fig. 4, days A10-A15 in Fig. 3). Therefore, we recommend the use of acquisition training with at least 24 trials, because it took that long before C57BL/6J mice demonstrated reliable use of the spatial search strategy.

Which measures of performance in the probe trial are most sensitive to detect impairments in visuo-spatial memory on the Barnes maze?

Average proximity to the escape hole and time in the correct zone on the LBM were slightly more sensitive measures of memory than visits to the correct hole. Therefore, we recommend the use of the percentage time in the correct zone or average proximity to the correct hole, rather than the percentage of hole visits. In the MWM, measures of probe trial performance differ in their sensitivity to detect memory impairment, with the average proximity to the escape platform being more sensitive than the percentage time in the correct quadrant or the frequency of platform crossings (Maei et al. 2009). The overall similarity in sensitivity of each measure of memory on the Barnes maze may occur because

Figure 7. Mean (+SEM) difference score reversal effects (R1 and RT2) for latency $(A)$, errors $(B)$, hole deviation scores $(C)$, and distance traveled $(D)$ on the SWBM, the SBM, and the LBM. (*) Significantly greater than zero; (\#) significantly different from mice trained with a random escape hole location. 

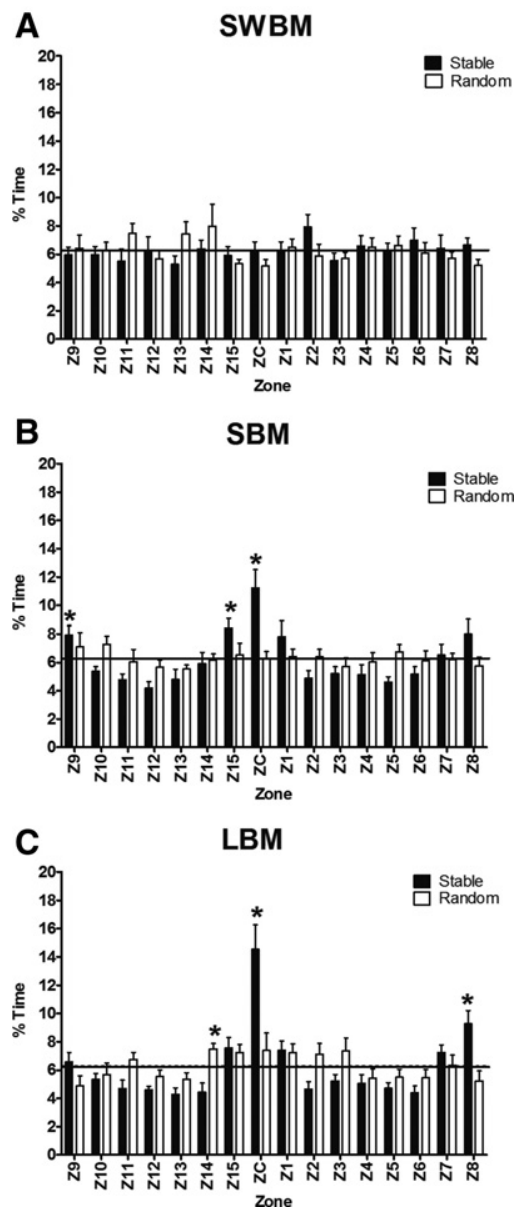

Figure 8. Mean percent time $(+S E M)$ in the correct zone $(Z C)$ and the other 15 zones $(Z 1-15)$ during the reversal probe trial with visible extra/ intra-maze cues following reversal training for mice trained with stable or random escape holes on the SWBM $(A)$, the SBM (B), and the LBM (C). Z8 indicates the location of the escape hole during the acquisition training phase. The horizontal line indicates chance level of performance $(6.25 \mathrm{sec})$ and asterisks $\left(^{*}\right)$ indicate when the time spent in a zone is greater than expected by chance $(P<0.05)$.

mice can remain stationary near the correct escape hole for extended time periods, whereas the requirement to maintain active swimming in the MWM increases the propensity for mice to swim away from the platform and leave the correct quadrant (Van Dam et al. 2006).

\section{What does the analysis of search strategies tell us about learning and memory on the Barnes maze?}

As mice learn to locate the escape hole location, their search strategies change based on the demand characteristics of the maze design. In all three mazes, mice used the random search strategy at the beginning of training (block 1) and then rapidly shifted to the more efficient serial search strategy on block 2. For mice trained with a random hole location, the serial search strategy was the predominant method for finding the escape hole on all three maze designs. When trained with a stable escape hole, however, mice on the SBM and LBM increased their use of the spatial strategy over blocks, and this increase was most evident on the LBM. In the SWBM, even when trained with a stable escape hole location, mice used the serial search strategy throughout acquisition and reversal training. We have previously suggested that the increased use of the serial strategy on the SWBM is due to the wall surrounding the maze, which promotes thigmotaxis and/or occludes extra-maze visual cues (O'Leary et al. 2011). Although the relative influence of each of these factors on performance is unknown, we do not expect that the spatial search strategy can be acquired with transparent walls, which do not occlude extramaze cues, as mice also did not use intra-maze visual cues to locate the escape hole on the SWBM design.

Our search path data (Fig. 4) show that mice learned to use the spatial search strategy over acquisition trials, but it was not used significantly more than the other strategies until the fourth and fifth blocks (18-30 trials). This indicates that with short training periods ( $<18$ trials) mice will not receive sufficient training to acquire the spatial strategy, and that measures of latency, errors, hole deviation scores, and distance traveled would not be accurate indicators of visuo-spatial learning, but instead reflect learning to use the serial strategy with increased efficiency. It appears that mice learn to use the most efficient search strategy for each test situation (stable vs. random), and that when visuo-spatial learning was prevented, mice trained with a random escape hole learned to use the serial search strategy efficiently and improved their performance by $65 \%-80 \%$ over acquisition training. Therefore, measures of extra-maze cue use and probe trials are necessary to demonstrate that mice are actually using extra-maze visual cues to learn a spatial search strategy on the Barnes maze. Search path analysis confirms that improved performance during acquisition training is due to increased use of visuo-spatial learning strategies and not due to the acquisition of nonspatial learning strategies.

\section{What does reversal training reveal about visuo-spatial learning and memory on the Barnes maze?}

Mice trained with a stable escape hole location on the SBM and LBM were impaired on the first day of reversal training, indicating that they learned the location of the acquisition escape hole, and searched at this location before finding the new escape hole. As expected, mice trained with a random escape hole location were not impaired on the reversal trials on any of the three maze designs. Although mice trained with a stable escape hole location on the SBM and LBM improved performance during reversal training, they reverted to a serial strategy and did not return to the level of performance shown on day A15. This indicates that five days of reversal training was not sufficient for mice to learn to use the spatial search strategy to navigate to the reversal escape hole. Interestingly, mice on the SBM and LBM remembered the former acquisition escape hole location as well as the new escape hole location during the reversal probe trial, indicating that memory for the previous escape hole location was not extinguished before memory for the novel escape hole location was acquired (O'Leary and Brown 2009, 2012).

\section{Does apparatus design determine the validity of the Barnes maze as a visuo-spatial learning and memory test for mice?}

The SWBM was the least valid maze design for visuo-spatial learning and memory because mice (1) did not learn to use the spatial search strategy, (2) the size of the reversal effects was small, and (3) memory performance was poor in probe trials with visible extra/ intra-maze cues and unimpaired when cues were blocked by a curtain. The poor spatial memory performance of mice on the SWBM occurs independently of the size of the zone around the correct hole or of the length of the probe trial (O'Leary and Brown 
2012), and is due to the use of the serial search strategy in which mice do not attend to visuo-spatial cues when searching for the escape hole and therefore do not learn the spatial location of the escape hole.

Mice on the SBM demonstrated inconsistent use of extramaze cues, because (1) they did not predominately use the spatial search strategy and (2) the size of the reversal effects was small. However, mice on the SBM did show good visuo-spatial memory performance in probe trials with visible extra-maze cues, and were impaired in the probe trial when visuo-spatial cues were blocked by a curtain. This demonstrates that mice remembered the location of the escape hole relative to extra-maze visual cues, but did not efficiently use this information to learn the location of the escape hole on the SBM. The LBM was the most valid maze design for visuo-spatial learning and memory because (1) mice learned to use the spatial search strategy, (2) the size of the reversal effects was large, and (3) memory performance was good in probe trials where visuo-spatial cues were available and impaired when cues were blocked by a curtain. Together these results indicate that mice on the LBM reliably use the extra-maze visual cues to locate the escape hole using a visuo-spatial strategy, replicating our previous results (O'Leary et al. 2011; O'Leary and Brown 2012).

\section{What about sex differences?}

Generally male rodents perform better on visuo-spatial learning and memory tasks than females (Jonasson 2005; Bettis and Jacobs 2009; Simpson and Kelly 2012), but very few sex differences have been reported in mice on the Barnes maze, and these differences are dependent on the apparatus design and test procedure used. We have previously found that males show better memory performance than females on the LBM, and have larger reversal effects than females during reversal training, due to increased perseveration at the previous location of the escape hole ( $\mathrm{O}^{\prime}$ Leary and Brown 2009, 2012). In the present study we replicated the sex difference in probe trial performance on the LBM, but not the sex differences in reversal effects, due to the high variability in the size of the reversal effects observed in female mice. These sex differences seem to be related to search strategy use. Males appear to learn to use the spatial strategy more rapidly and effectively than females, resulting in a better probe trial performance, and causing males to persevere longer than females at the original escape hole during reversal trials (O'Leary and Brown 2012). It should be noted, however, that the statistical power to detect interactions involving sex was low in this study due to low sample sizes ( $N=6$ per sex in each training condition), and therefore additional sex differences may be present but not detected. Further research is required to determine the reliability of these sex differences and the mechanisms underlying them.

\section{Should apparatus design and test procedure on the Barnes maze be standardized?}

This experiment has identified many experimental parameters that influence the validity and sensitivity of the Barnes maze as a test of visuo-spatial learning and memory. However, the present experiment used C57BL/6J mice, and the recommended apparatus design and test procedure may not be optimal for other mouse strains, such as 129S1/SvImJ and A/J, which show low levels of exploration on the Barnes maze (Holmes et al. 2002; O'Leary et al. 2011). For example, DBA/2J mice perform better in a modified Barnes maze with holes distributed throughout the edge and center of the maze and when appetitive food reward is used than on a Barnes maze design with holes around the edge and aversive stimuli (Youn et al. 2012). Furthermore, for some strains the optimal apparatus design and test procedure for the Barnes maze may be less valid and sensitive than other visuo-spatial tests, and in this case those tests should be preferentially used over the Barnes maze. Therefore, the proposal of an optimal set of experimental parameters for inter-laboratory standardization can only be justified once data from different strains and laboratories are compared. However, we propose that experimental parameters that increase the sensitivity for detecting performance differences should be used whenever possible, and that the continued use of suboptimal experimental parameters must be justified before their use in future research.

\section{General conclusions}

This experiment demonstrated that the LBM was the most valid and sensitive Barnes maze design for detecting visuo-spatial learning and memory impairment in mice. With respect to the measures of performance, latency showed low sensitivity compared to that of errors, hole deviation scores, and distance traveled. Furthermore, the primary measures of performance were more sensitive than the total measures of performance, because primary measures controlled for delays to enter the escape hole. Search path analysis provided a measure of the rate at which the spatial search strategy was learned over training trials. Since mice learned to use the spatial search strategy over the first 10-12 d, training should involve a minimum of 20-24 training trials before the memory probe trials are given. Percentage time in the correct zone and average proximity to the correct escape hole were more sensitive measures of memory than percentage visits to the correct hole. Therefore, we recommend that future research using the Barnes maze for mice (1) uses larger maze designs, (2) uses multiple primary measures of learning and memory performance, (3) records search paths, and (4) includes at least 24 training trials so that the effects on visuo-spatial learning and memory can be reliably detected. Furthermore, the use of (5) reversal training and (6) probe trials to test memory should be included to establish that mice are using extra-maze cues to locate the escape hole.

These results have significant implications for studies on the neural and genetic bases of visuo-spatial learning and memory. For example, suboptimal apparatus design and test procedures may not have sufficient sensitivity to detect the effects of lesions, or genetic or pharmacological manipulations on hippocampaldependent visuo-spatial learning and memory. Furthermore, memory systems can be dissociated, as hippocampal lesions disrupt spatial but not procedural memory (Packard and McGaugh 1996; White and McDonald 2002). Therefore, hippocampal lesions would be expected to disrupt the acquisition of visuo-spatial memories (spatial strategy) while leaving procedural memory and acquisition of the serial strategy intact. Impairments in acquisition of the serial strategy may also result in impairments on the Barnes maze, but may be due to disruption of brain regions involved in nonhippocampal learning and memory (e.g., dorsal striatum). Moreover, mice are proficient in switching strategies when visuo-spatial information is no longer reliable, given that performance improved rapidly as mice shifted from the visuo-spatial to the procedural strategies in reversal learning. Thus experiments on the neural, genetic, and pharmaceutical correlates of visuospatial learning and memory must ensure that the behavioral data support the claim that mice are, in fact, using visuo-spatial processes for learning and memory. It has been known for over 50 years that there is more than one type of learning (Tolman 1949) and that rodents can learn both spatial and procedural strategies (Tolman et al. 1946; Restle 1957). This experiment has shown that mice switch between spatial and nonspatial strategies 
to navigate efficiently to the escape hole on the Barnes maze, depending on the apparatus design and test procedure.

\section{Materials and Methods}

\section{Subjects}

Male $(N=36)$ and female $(N=36) \mathrm{C} 57 \mathrm{BL} / 6 \mathrm{~J}$ mice at $8 \mathrm{wk}$ of age were obtained from the Jackson Laboratories (Bar Harbor, Maine) and housed in same-sex groups of four in polyethylene cages $(35 \times 12 \times 12 \mathrm{~cm})$ with pine chip bedding and a polyvinyl chloride tube $(5-\mathrm{cm}$ diameter $\times 8-\mathrm{cm}$ length) for enrichment. Food (Purina rodent chow, no. 5001) and tap water were available ad libitum. Mice were housed under a reversed 12:12 h light-dark cycle (lights off at 9:30 am) and behavioral tests were completed during the dark phase of the light-dark cycle. Starting when they were $14 \mathrm{wk}$ of age, all mice were tested in the open-field, elevated plus maze, and light-dark box (data to be included in a separate manuscript) before being tested in the Barnes maze. Experimental procedures were approved by the Dalhousie University Committee on Animal Care.

\section{Barnes maze designs}

Three versions of the Barnes maze were used: (1) the small-walled Barnes maze, (2) the small Barnes maze, and (3) the large Barnes maze, as depicted in O'Leary and Brown (2012). The small-walled Barnes maze (SWBM) was constructed from white polyethylene and consisted of a circular open arena $(69 \mathrm{~cm}$ in diameter) elevated to $48.4 \mathrm{~cm}$ by four wooden legs. There were 16 circular holes ( $4.45 \mathrm{~cm}$ in diameter) equally spaced around the perimeter, and positioned $1.3 \mathrm{~cm}$ from the edge of the maze. A white wall (15 cm high) circled the edge of the maze. Four intra-maze cues, consisting of colored (red, blue, black, and orange) shapes $(8 \mathrm{~cm}$ high) made of Bristol board were placed inside plastic sleeves $(9 \times 9 \mathrm{~cm})$ adhered to the maze wall. The intra-maze cues were positioned equal distances from each other and no cue was located directly over an escape hole. A plastic escape box $(13 \times 29 \times$ $14 \mathrm{~cm}$ ) with a metal ramp was positioned beneath one of four holes $(4,8,12$, or 16$)$. The escape box and ramp were painted black and the area of the ramp that declined into the escape box was covered with wire mesh $(1 \mathrm{~cm} \times 1 \mathrm{~cm})$. As described by Poucet et al. (1991), to reduce shadows a black cloth curtain was draped from the maze floor and covered the area below the maze.

Two 150-W flood lamps positioned $155 \mathrm{~cm}$ above the maze and a buzzer $(1-37.2 \mathrm{kHz}, 89$ decibels) hung $15 \mathrm{~cm}$ above the maze were used as aversive stimuli. A blue polyvinyl chloride start tube $(12.5 \mathrm{~cm}$ high, $8 \mathrm{~cm}$ in diameter) was raised from the center of the maze using a system of pulleys. The LimeLight (Actimetrics) tracking system, on an IBM PC computer and a SONY video camera (model SSC-M183), was used to record (5 frames/sec) the movement of mice on the SWBM. Distal extra-maze visual cues consisted of objects within the room (table, computer, sink, door, etc.), the experimenter, the geometric properties of the testing room $(2.6 \mathrm{~m} \times 5.6 \mathrm{~m})$, and shapes (triangle, circle, and rectangle) placed on the walls.

The small Barnes maze (SBM) was a smaller version of the Barnes maze originally designed for rats and was identical to the SWBM except there was no wall and no intra-maze visual cues around the edge. The large Barnes maze (LBM) was constructed from white polyethylene and was identical in diameter $(122 \mathrm{~cm})$ to the original design for rats (Barnes 1979), but had 16 holes $(4.5 \mathrm{~cm}$ in diameter) rather than 18 holes. Sixteen holes were used so that the number of holes in the three Barnes maze designs was the same. Mice were tested on the SBM and the LBM in the same room using the same escape box, extra-maze cues, and aversive stimuli as described for the SWBM.

\section{Procedure}

Mice were tested in squads of four, and between trials they were placed into individual holding cages with pro-chip bedding (P.W.I. Industries Inc.). Half of the mice tested on each maze were trained with an escape hole located in a stable spatial loca- tion across trials (stable condition). To reduce intra-maze odor cues, each of the four mice within a squad were assigned a different escape hole location for the duration of testing. The other half of the mice were trained with an escape hole randomly located at different spatial locations on each trial and test day (random condition). The location of the escape hole was determined by randomly selecting one of the holes $(1-16)$ to be placed at spatial location " $\mathrm{A}$ " across trials (one sequence was used for all mice). The escape box could then be placed randomly beneath holes 4 , 8,12 , or 16 for each trial. As a result, the location of the escape hole varied across 16 different spatial locations (A-P). To prevent mice in the random condition from using extra/intra-maze visual cues, the escape hole was not in the same location for more than two consecutive trials for any mouse.

Mice in each condition completed five phases of testing: habituation, acquisition training, acquisition probe, reversal training, and reversal probe. Mice received one habituation trial in which they were placed beside their respective escape hole underneath an inverted 2-L transparent glass beaker for $5 \mathrm{~min}$. This procedure allowed mice to experience the aversive bright lights above the Barnes maze and to practice descending into the escape box. During the habituation trial, intra-maze cues were removed and a curtain was placed around the maze to block extra-maze cues so that learning the escape hole's location relative to extra/intramaze cues could not occur.

The acquisition phase consisted of 15 training days (A1-A15) with two trials per day. On each trial a mouse was placed into the start tube located in the center of the maze, the start tube was raised, and the buzzer was turned on until the mouse entered the escape hole. After each trial mice remained in the escape box for $\sim 30 \mathrm{sec}$ before being returned to their holding cage, which was placed in a dark room adjacent to the test room for the inter-trial interval (5-10 min). For each training, trial mice were given $5 \mathrm{~min}$ to locate the escape hole, after which they were guided to the escape hole and coaxed to enter it using a plastic container. Mice that still failed to enter the escape hole were placed directly into the escape box. If a mouse climbed back onto the maze after entering the escape box, the buzzer was turned on until it re-entered the escape box. A mouse that fell down an incorrect hole or climbed down the curtain below the maze was returned to its holding cage, and the trial was re-run after all the other mice within the squad had completed the respective training trial.

During training, mice may fail to enter the escape box when they locate the escape hole. Therefore we scored behaviors until the mice first located the escape hole (primary performance) and until the mice entered the escape hole (total performance) (Harrison et al. 2006). During acquisition training, five measures of learning performance were recorded: (1) the latency to locate/enter the escape hole, (2) number of errors, (3) hole deviation scores, (4) distance traveled before locating the escape hole, and (5) search paths. Latency was the time required to locate/enter the escape hole. An error occurred when a mouse dipped its head into a hole that did not provide escape. Multiple consecutive head-dips into a single hole were recorded as a single error. The hole deviation score was the number of escape holes between the hole the mouse first investigated (touched or passed its head over) and the correct escape hole (range $=1-8$ ). Distance traveled was the length of the path which the animal took before locating/ entering the escape hole.

The search paths were categorized into random, serial, and spatial, as described by Barnes (1979), using data recorded with the Limelight software or from video tapes. A random search path was scored if a mouse displayed a nonsystematic search with multiple crossings through the center of the maze. Trials in which animals did not locate the escape hole were considered a random search. A serial search path was scored if a mouse moved around the edge of the maze past at least three adjacent holes before entering the escape hole. A spatial search path was scored when a mouse moved directly toward the escape hole from the center of the maze, and did not approach a hole more than two holes away from the correct hole. During reversal training, some mice moved directly to the acquisition escape hole, before 
moving directly to the opposite side of the maze to enter the reversal escape hole, and these trials were scored as spatial search strategies. Search strategy was analyzed using the percentage of trials on which each strategy was used in blocks of $3 \mathrm{~d}$ (six trials) during acquisition for each of the three maze designs. Search strategy was scored only for the last three days of reversal training (R3-R5).

Between training trials the maze floor and the escape box were cleaned with Sparkleen (Fisher Scientific Co.) mixed with water, and between each squad of mice the escape box was rinsed with water. For mice trained with a stable escape hole location, the maze was rotated $90^{\circ}$ between trials so that odor cues on the maze floor did not predict the escape hole location. After rotating the SWBM between trials, the intra-maze visual cues were moved to their original spatial locations.

The acquisition probe trial phase was four days long. A probe trial with visible intra- and/or extra-maze cues available, but no buzzer and no escape box present, was completed on the day following the acquisition phase. The next day mice completed two re-training trials with the escape box present to reduce extinction that might have occurred during the probe trial. On the third day, the second probe trial with a curtain placed around the maze to block extra-maze cues was completed to determine if mice could locate the escape hole in the absence of the extra-maze visual cues. Intra-maze cues were removed from the SWBM during this probe trial. The curtain was attached to a circular metal rod $2 \mathrm{~m}$ above the maze, and the bottom of the curtain hung $10 \mathrm{~cm}$ below the maze floor. The curtain hung $26 \mathrm{~cm}$ away from the edge of the SBM and SWBM, and directly beside the edge of the LBM. On the fourth day an additional two re-training trials were completed. During the probe trials, memory was assessed using two procedures. First, the Limelight software was used to divide the maze into 16 virtual pie-shaped zones, with one zone per hole. The time spent in each of the 16 zones was recorded and visuo-spatial memory was assessed by the time the mouse spent in the zone that contained the escape hole during the previous training phase. For mice trained with a random escape hole, the correct hole was defined as the one which provided escape on the last training trial completed. Second, using EthoVision (Noldus) software, circular zones were created around each hole, and memory was assessed using the number of entries mice made into each zone and the average proximity (i.e., distance in centimeters) of the mouse to each of the holes over the 5-min probe trial.

The reversal training phase consisted of five training days (R1-R5) with two trials per day. In this phase, for mice trained with a stable escape hole the location of the escape hole was moved $180^{\circ}$ from its location during the acquisition training phase. For mice trained with a random escape hole, the escape hole continued to be positioned randomly at different spatial locations. The reversal effect was assessed with latency, error, hole deviation, and distance traveled difference scores, calculated by subtracting the mean performance on the last re-training day of the acquisition probe phase (RT-2) from the performance on the first day of reversal training (R1). A 5-min reversal probe trial was given the day after the reversal phase to assess visuo-spatial memory for the new escape hole location. Memory was assessed by the time spent in each of the 16 zones, as described for the acquisition probe trial.

\section{Data analyses}

Data were analyzed separately for each maze using StatView (ver. 5.0.1, SAS Institute Inc.) and SPSS software (ver. 20, IBM). Because very few sex differences were significant (see Supplemental results 1 ), data were collapsed over sex. The mean for each measure of learning performance was calculated across the two trials for each day and these daily means were analyzed with mixed design ANOVAs with training procedure (stable vs. random) as a between-subject factor and day as a within-subject factor. For within-subject effects and interactions, Mauchly's test for the sphericity of variance was used, and when sphericity was violated, the Greenhouse-Geisser correction to degrees of freedom was used (Harrison et al. 2006). Therefore, although the same number of animals were used in each analysis, the degrees of freedom for within-subject effects and interactions were not identical. Analyses of search strategies were completed separately for each maze design using Mann-Whitney $U$-tests with training procedure as a between-subject factor for each 3-d training block. Friedman one-way ANOVA tests were also completed to determine if search-strategy use changed over acquisition training. For reversal effects and probe trial measures, one-way ANOVAs were completed with training procedure as a between-subject factor. $t$-tests were used to determine whether reversal effects were greater than zero and whether the percent time in each zone was greater than chance (6.25\%). In order to determine the power/sensitivity to detect differences in learning and memory in mice given different training procedures, effect size $\left(\eta^{2}\right)$ and power $(P w, \alpha=0.05)$ for the training condition effect are reported for each measure of learning and memory during the acquisition and probe trials. Effect size was also calculated for cumulatively longer training periods (1-15 d) for each measure of learning, in order to determine how effect size changes as a function of number of training days. Post-hoc tests were completed with simple effects analyses and Student-Newman-Keuls comparisons $(P<0.05)$. Only significant interactions are reported. We also analyzed acquisition training, reversal training, and probe trial data with both maze design and training condition as between-subject factors to confirm if the maze by training condition interactions were significant and these results are found in the Supplemental results.

\section{Acknowledgments}

We thank Bud Eisner for constructing the Barnes mazes and Chris Wright and Andrew Johnston for technical assistance with equipment used in this experiment. Also, we thank Dr. Leslie Phillmore, Dr. Vincent LoLordo, and Dr. Heather Schellinck for useful comments concerning experimental design. Data was collected with the help of Antonius Diab, Ana Correa, Mark Bartolacci, and Kevin Le. This research was completed with funding provided by an NSERC discovery grant to R.E.B. and a NSHRF graduate student research award to T.P.O.

\section{References}

Alberto CS, Walsh K, Davisson MT. 1999. Motor dysfunction in a mouse model for Down syndrome. Physiol Behav 68: 211-220.

Bach ME, Hawkins RE, Osman M, Kandel ER, Mayford M. 1995. Impairment of spatial but not contextual memory in CaMKII mutant mice with a selective loss of hippocampal LTP in the range of the $\theta$ frequency. Cell 81: 905-915.

Barnes CA. 1979. Memory deficits associated with senescence: A neurophysiological and behavioral study in the rat. J Comp Physiol Psychol 93: 74-104.

Bettis TJ, Jacobs LF. 2009. Sex-specific strategies in spatial orientation in C57BL/6J mice. Behav Processes 32: 249-255.

Blokland A, Geraerts E, Been M. 2004. A detailed analysis of rats' spatial memory in a probe trial of a Morris task. Behav Brain Res 154: 71-75.

Cahill L, McGaugh JL, Weinberger NM. 2001. The neurobiology of learning and memory: Some reminders to remember. Trends Neurosci 24: $571-581$.

Crabbe JC, Wahlsten D, Dudek BC. 1999. Genetics of mouse behaviour: Interactions with laboratory environment. Science 284: 1670-1672.

Crusio WE. 1999. Methodological considerations for testing learning in mice. In Handbook of molecular-genetic techniques for brain and behavior research Vol. 13 (ed. WE Crusio, RT Gerlai), pp. 638-651. Elsevier, Amsterdam.

Crusio WE, Schwegler H. 2005. Learning spatial orientation tasks in the radial-maze and structural variation in the hippocampus in inbred mice. Behav Brain Funct 1: 3 .

Gage FH, Dunnett SB, Blörklund A. 1984. Spatial learning and motor deficits in aged rats. Neurobiol Aging 5: 43-48.

Gallagher M, Burwell R, Burchinal M. 1993. Severity of spatial learning impairment in aging: Development of a learning index for performance in the Morris water maze. Behav Neurosci 107: 618-626.

Garcia MF, Gordon MN, Hutton M, Lewis J, McGowan E, Dickey CA, Morgan D, Arendash GW. 2004. The retinal degeneration (rd) gene seriously impairs spatial cognitive performance in normal and Alzheimer's transgenic mice. Neuroreport 15: 73-77. 
Harrison FE, Reiserer RS, Tomarken AJ, McDonald MP. 2006. Spatial and nonspatial escape strategies in the Barnes maze. Learn Mem 13: 809-819.

Holmes A, Wrenn CC, Harris AP, Thayer KE, Crawley JN. 2002. Behavioral profiles of inbred strains on novel olfactory, spatial and emotional tests for reference memory in mice. Genes Brain Behav 1: 55-69.

Iivonen H, Nurminen L, Harri M, Tanila H, Puoliväli J. 2003.

Hypothermia in mice tested in the Morris water maze. Behav Brain Res 141: 207-213.

Jonasson Z. 2005. Meta-analysis of sex differences in rodent models of learning and memory: A review of behavioral and biological data. Neurosci Biobehav Rev 28: 811-825.

Koopmans G, Blokland A, van Nieuwenhuijzen P, Prickaerts J. 2003. Assessment of spatial learning abilities of mice in a new circular maze. Physiol Behav 79: 683-689.

Levin ED. 2001. Use of the radial arm maze to assess learning and memory in rodents. In Methods of behavior analysis in neuroscience (ed. JJ Buccafusco), pp. 189-199. CRC Press, Boca Raton, FL.

Maei HR, Zaslavsky K, Teixeira CM, Frankland PW. 2009. What is the most sensitive measure of water maze probe test performance? Front Integr Neurosci 3: 1-9.

Miyakawa T, Yared E, Ho Pak J, Huang FL, Huang K, Crawley JN. 2001. Neurogranin null mutant mice display performance deficits on spatial learning tasks with anxiety related components. Hippocampus 11: $763-775$.

Morris R. 1984. Development of a water-maze procedure for studying procedural learning in the rat. J Neurosci Meth 11: 47-60.

Nguyen PV, Abel T, Kandel ER, Bourtchouladze R. 2002. Strain dependent differences in LTP and hippocampus-dependent memory in inbred mice. Learn Mem 7: 170-179.

O'Leary TP, Brown RE. 2009. Visuo-spatial learning and memory deficits on the Barnes maze in the 16-month-old APPswe/PS1dE9 mouse model of Alzheimer's disease. Behav Brain Res 201: 120-127.

O'Leary TP, Brown RE. 2012. The effects of apparatus design and test procedure on learning and memory performance of $\mathrm{C} 57 \mathrm{BL} / 6 \mathrm{~J}$ mice on the Barnes maze. J Neurosci Meth 30: 315-324.

O'Leary TP, Savoie V, Brown RE. 2011. Learning, memory and search strategies of inbred mouse strains with different visual abilities on the Barnes maze. Behav Brain Res 216: 531-542.

Packard MG, McGaugh JL. 1996. Inactivation of hippocampus or caudate nucleus with lidocaine differentially affects expression of place and response learning. Neurobiol Learn Mem 65: 65-72.

Pompl PN, Mullan MN, Bjugstad K, Arendash GW. 1999. Adaptation of the circular platform spatial memory task for mice: Use in detecting cognitive impairment in the $\mathrm{APP}_{\mathrm{sw}}$ transgenic mouse model for Alzheimer's disease. J Neurosci Meth 87: 87-95.

Poucet B, Herrmann T, Buhot MC. 1991. Effects of short-lasting inactivations of the ventral hippocampus and medial septum on long-term and short-term acquisition of spatial information in rats. Behav Brain Res 44: 53-65.

Restle F. 1957. Discrimination of cues in mazes: A resolution of the "place-vs.-response" question. Psych Rev 64: 217-228.

Simpson J, Kelly JP. 2012. An investigation of whether there are sex differences in certain behavioural and neurochemical parameters in the rat. Behav Brain Res 229: 289-300.

Stewart S, Cacucci F, Lever C. 2011. Which memory task for my mouse? A systematic review of spatial memory performance in the Tg2576 mouse model of Alzheimer's disease. J Alzheimers Dis 26: $105-126$.

Tolman EC. 1949. There is more than one kind of learning. Psych Rev 56: $144-155$.

Tolman EC, Ritchie BF, Kalish D. 1946. Studies in spatial learning. II. Place learning versus response learning. J Exp Psych 35: 221-229.

Van Dam D, Lenders G, De Deyn PP. 2006. Effect of Morris water maze diameter on visual-spatial learning in different mouse strains. Neurobiol Learn Mem 85: 164-172.

Wahlsten D. 2011. Task refinement and standardization. In Mouse behavioural testing: How to use mice in behavioural neuroscience, pp. 215-233. Academic Press, Burlington, MA.

Wahlsten D, Metten P, Phillips TJ, Boehm SL II, Burkhart-Kasch S, Dorow J, Doerksen S, Downing C, Fogarty J, Rodd-Henricks K, et al. 2003. Different data from different labs: Lessons from studies of gene-environment interaction. J Neurobiol 54: 283-311.

Wenk GL. 2004. Assessment of spatial memory using the radial arm maze and Morris water maze. Curr Protoc Neurosci Unit 8.5 A.

White NM, McDonald RJ. 2002. Multiple parallel memory systems in the brain of the rat. Neurobiol Learn Mem 77: 125-184.

Yassine N, Lazaris A, Dorner-Ciossek C, Després O, Meyer L, Maitre M, Mensah-Nyagan AG, Cassel J-C, Mathis C. 2013. Detecting spatial memory deficits beyond blindness in tg2576 Alzheimer mice. Neurobiol Aging 34: 716-730.

Youn J, Ellenbroek BA, van Eck I, Roubos S, Verhage M, Stiedl O. 2012. Finding the right motivation: Genotype-dependent differences in effective reinforcements for spatial learning. Behav Brain Res 226: $397-403$.

Received August 7, 2012; accepted in revised form November 20, 2012. 


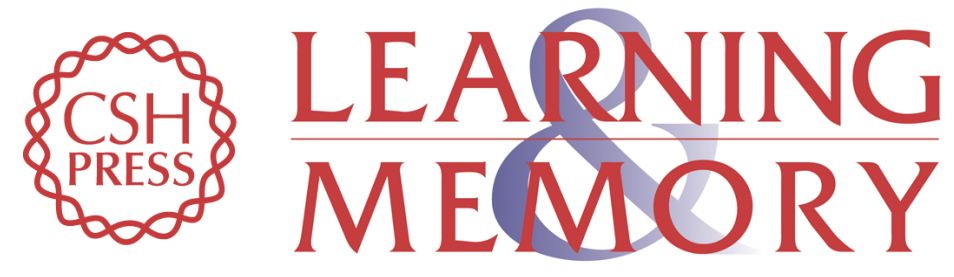

\section{Optimization of apparatus design and behavioral measures for the assessment of visuo-spatial learning and memory of mice on the Barnes maze}

Timothy P. O'Leary and Richard E. Brown

Learn. Mem. 2013, 20:

Access the most recent version at doi:10.1101/lm.028076.112

Supplemental Material

References License

Email Alerting Service
http://learnmem.cshlp.org/content/suppl/2013/01/07/20.2.85.DC1

This article cites 37 articles, 2 of which can be accessed free at: http://learnmem.cshlp.org/content/20/2/85.full.html\#ref-list-1

Receive free email alerts when new articles cite this article - sign up in the box at the top right corner of the article or click here. 